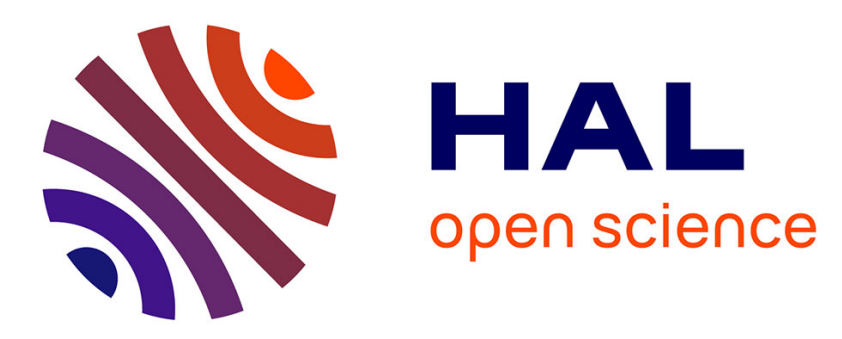

\title{
Numerical Investigation of an Academic Mistuned Bladed Disk Dynamics Accounting for Blade/Casing Contacts
}

Jeanne Joachim, Florence Nyssen, Alain Batailly

\section{To cite this version:}

Jeanne Joachim, Florence Nyssen, Alain Batailly. Numerical Investigation of an Academic Mistuned Bladed Disk Dynamics Accounting for Blade/Casing Contacts. ASME 2019 International Mechanical Engineering Congress and Exposition, Nov 2019, Salt Lake City, United States. 10.1115/IMECE201910300 . hal-02884585

\section{HAL Id: hal-02884585 \\ https://hal.science/hal-02884585}

Submitted on 30 Jun 2020

HAL is a multi-disciplinary open access archive for the deposit and dissemination of scientific research documents, whether they are published or not. The documents may come from teaching and research institutions in France or abroad, or from public or private research centers.
L'archive ouverte pluridisciplinaire HAL, est destinée au dépôt et à la diffusion de documents scientifiques de niveau recherche, publiés ou non, émanant des établissements d'enseignement et de recherche français ou étrangers, des laboratoires publics ou privés. 


\title{
Numerical Investigation of an Academic Mistuned Bladed Disk Dynamics Accounting for Blade/Casing Contacts
}

\author{
Jeanne Joachim ${ }^{1}$, Florence Nyssen ${ }^{1}$, Alain Batailly ${ }^{1}$
}

\begin{abstract}
This contribution focuses on the combined analysis of mistuning and unilateral blade-tip/casing contacts. A 2D phenomenological finite element model of an aircraft engine fan stage is considered. It is reduced by means of the Craig-Bampton component mode synthesis method and contact treatment relies on a Lagrange multiplier algorithm within an explicit time-integration scheme. Blade-tip/casing contacts are initiated through the deformed shape of a perfectly rigid casing. Mistuning is accounted for on the blades only. Monte Carlo simulations are carried out in both linear and nonlinear configurations, which allows to compare amplifications predicted in both context due to mistuning. Following a thorough convergence analysis of the proposed numerical strategy, the influence of mistuning level as well as the configuration of the external forcing are investigated. Presented results underline the detrimental consequences of mistuning in a nonlinear structural context, yielding even higher vibration amplifications than in a linear context. A cross-analysis between linear and nonlinear computations reveals that no correlation is found between linear and nonlinear amplifications which suggests that the effect of existing strategies to mitigate vibration amplifications within a linear context may not be suitable within a nonlinear context.
\end{abstract}

Keywords

mistuning; nonlinear dynamics; rotor/stator interaction; stochastic dynamics; unilateral contact constraints

1 - Department of Mechanical Engineering, École Polytechnique de Montréal, P.O. Box 6079, Succ. Centre-Ville, Montréal, Québec, Canada H3C 3A7 


\title{
Enquête numérique sur le comportement vibratoire d'un modèle de roue aubagée désaccordée avec interfaces de contact aubes/carter
}

\author{
Jeanne Joachim ${ }^{1}$, Florence Nyssen ${ }^{1}$, Alain Batailly ${ }^{1}$
}

Résumé

Cet acte de conférence présente une analyse combinée des effets du désaccordage et d'interactions de contact sur le comportement vibratoire d'une roue aubagée académique. Un modèle éléments finis plan en deux dimensions de la soufflante d'un moteur d'avion est utilisé pour cette étude. Ce modèle éléments finis est réduit à l'aide d'une méthode de synthèse modale et le traitement du contact est opéré via un algorithme reposant sur le calcul de multiplicateurs de Lagrange au sein d'une procédure d'intégration temporelle de type différences finies centrées. Le contact aube/carter est initié par une déformation du carter supposé parfaitement rigide. Le désaccordage est pris en compte uniquement au niveau des aubes et des simulations de type Monte Carlo sont effectuées dans un contexte linéaire (réponse forcée à un chargement aérodynamique simplifié) et non-linéalire (réponse vibratoire au contact). Une attention particulière est portée sur la validation des résultats obtenus en s'assurant notamment de la convergence stochastique, par des indicateurs quantitatif et qualitatif. Les résultats obtenus attestent de la représentativité du modèle étudié par rapport aux standards de la littérature dans un contexte linéaire. Par ailleurs, lorsque le contact aubes/carter est pris en compte, les résultats obtenus montrent une augmentation significative des amplifications de vibration par rapport à celles observées dans un contexte linéaire. Une analyse croisée des résultats linéaire et non-linéaire souligne l'absence de corrélation entre les motifs engendrant les plus grandes amplifications linéaire et non-linéaire. Cette dernière observation suggère que les méthodes existantes pour réduire l'amplification des vibrations dans un contexte linéaire pourraient ne pas être adaptées à la réduction des amplifications dans le cas de contacts aubes/carter.

Mots-clés

désaccordage; dynamique non-linéaire; interactions rotor/stator; dynamique stochastique; contact unilatéral

1 - Département de génie mécanique, École Polytechnique de Montréal, P.O. Box 6079, Succ. Centre-Ville, Montréal, Québec, Canada H3C 3A7 


\section{INTRODUCTION}

Many research efforts have been dedicated to the analysis of bladed disk mistuning [1], a phenomenon that characterizes the unavoidably non-perfectly symmetric shape of aircraft engine rotating components, be it due to manufacturing tolerances or material inhomogeneities. Contrary to perfectly symmetric structures, mistuned bladed disks feature frequency split that impact their dynamic properties. Ever since the identification of localization phenomena related to mistuning and their detrimental effects due to high cycle fatigue [1], industry-driven research has focused on mitigating mistuning undesired consequences on bladed components' forced response. Within this linear context, a wide variety of solutions have been investigated such as the development of novel blade designs [2] and intentional mistuning patterns with optimized blade arrangements [3,4]. Very recently, researchers started to account for mistuning within nonlinear contexts such as the analysis of flutter interactions [5, 6] or blade root friction damping issues [7]. Latest researches suggest that intentional mistuning can even be used as a way to improve further modern aircraft engine designs [8]. In both linear and nonlinear contexts, robust numerical predictions accounting for a bladed disk mistuning are typically achieved by means of Monte Carlo simulations [9] or polynomial chaos expansion [10].

As the drive towards increased overall engine efficiency motivates engineers to reduce operating clearances, modeling the nonlinear vibrations subsequent to blade/casing structural contacts is of primary interest for aircraft engine manufacturers [11]. Yet the robustness of numerical predictions is still to be assessed with respect to a certain level of mistuning, as it may broaden the scope of investigation by altering the bladed disk dynamics.

To the best of the authors' knowledge, this paper presents the first analysis of the influence of small mistuning on a bladed disk nonlinear response following blade-tips/casing unilateral contacts. Stochastic analyses are conducted based on well-established methods for contact treatment and mistuning implementation applied on a $2 \mathrm{D}$ phenomenological finite element (FE) model of a civil aircraft engine fan stage. The model, mistuning implementation and contact numerical treatment are presented in the first section of the article. The second section then focuses on a thorough validation of the proposed methodology for nonlinear contact simulations on a given mistuned bladed disk. Presented results' robustness and numerical relevance are assessed through key parameters. In the third section, convergence of the stochastic simulations is assessed and nonlinear stochastic results are presented and analyzed with regards to linear stochastic results.

\section{MODELING}

The 2D phenomenological FE model used in this study is depicted in Fig. 1a. The blades are made of straight beam elements with 3 degrees of freedom (dof) per node whereas the disk is made of both straight and curved beam elements, these last featuring 4 dof per node. The model dimensions and properties, summarized in Tab.1, are adjusted so that the first bending modes normal frequencies are in the vicinity of $90 \mathrm{~Hz}$. This model is similar to the one used in previous publications dedicated to a variety of contact phenomena for a tuned bladed disk [12, 13] where it was found to be fairly representative of an actual aircraft engine fan stage. It should also be underlined that the proposed methodology, though applied to a 2D model, is inherently applicable to 3D industrial models.

\section{Modal reduction}

The Craig-Bampton component mode synthesis method [14] is used to reduce the dimension of the model and thus lower its computational cost. This reduction technique advantageously yields a mixed reduced set of coordinates in which physical dof can be kept. This way, displacements in both directions of space at the tip of each of the 12 blades are retained within the reduced order model (ROM) for efficient contact treatment. These 24 boundary dof, see Fig. 1a, are complemented by $\eta$ modal dof so that the ROM properly represents the initial finite element model. The SAFE diagram for both the ROM and the full-FE model is depicted in Fig. 2. Modes of the first bending mode (1B) are closely spaced in frequency, from $90.1 \mathrm{~Hz}$ to $93.2 \mathrm{~Hz}$

\section{Contact treatment}


Table 1. Geometrical parameters and material properties of the finite element model.

\begin{aligned} \hline number of blades & $N=12 \\$\hline tip radius & $50 \mathrm{~cm} \\$\hline hub radius & $20 \mathrm{~cm} \\$\hline blade discretization & 5 straight beams \\ \hline nominal Young's modulus & $E_{0}=2.1 \cdot 10^{11} \mathrm{~Pa} \\$\hline density & $7800 \mathrm{~kg} / \mathrm{m}^{3} \\$\hline modal damping ratio & $5 \cdot 10^{-3} \\$\hline Poisson's ratio & 0.3 \\ \hline & \end{aligned}

\section{Contact initiation}

In this study, contact is initiated by a deformation of the surrounding rigid casing, see Fig. $1 \mathrm{~b}$ and Fig. 3. For the sake of simplicity, the number of privileged contact areas on the casing is here defined as $n_{d}$, in analogy with the number of nodal diameters of free vibration modes that would be excited by such configuration for the tuned system. Each of the $n_{d}$ casing bumps are evenly spaced along the casing circumference. A smooth evolution of the contact surface is achieved through an exponential growth of the bumps in the first $0.01 \mathrm{~s}$ of the simulation in order to avoid any initial blade/casing penetration. The variation of the casing radius due to these bumps is referred to as the casing radial deformation amplitude $c_{\mathrm{rd}}$. Each bump is defined using the function:

$f(\theta)=-c_{\mathrm{rd}} \exp \left(-\left(\frac{\theta}{0.1 \frac{2 \pi}{n_{d}}}\right)^{2}\right)$

As depicted in Fig. 3, contact may occur when $c_{\mathrm{rd}}$ is higher than the initial blade/casing clearance.

Numerical solution

Within the small perturbation framework, the equation of motion to be solved in the time domain is:

$\mathbf{M u}+\mathbf{D} \dot{\mathbf{u}}+\mathbf{K u}=\mathbf{F}(t)+\mathbf{F}_{\mathrm{nl}}(\mathbf{u}, t)$

where $\mathbf{M}, \mathbf{D}$ and $\mathbf{K}$ are respectively the mass, damping and stiffness matrices, $\mathbf{u}$ denote the generalized displacements vectors and $\dot{\mathbf{u}}$, $\mathbf{u}$ respectively their first and second order time derivatives, $\mathbf{F}$ and $\mathbf{F}_{\mathrm{nl}}$ are the linear and nonlinear excitation force vectors. The forward increment Lagrange multiplier method [15] is employed to compute the solution of Eq. (2). The associated prediction/correction algorithm is used similarly to what was previously described in [12].

Mistuning

Implementation

Small mistuning is implemented through material properties variations, thus echoing with tolerances formulation of industrial manufacturing processes. As blade mistuning has been shown to be critically dominant $[16,1]$, mistuning is here considered as a blade Young's modulus variation for each blade $i$ :

$E_{i}=E_{0}\left(1+(\delta E)_{i}\right) \quad, \quad i \in[1, N]$

with $E_{0}$ the nominal Young's modulus of the tuned system. The Young's modulus variation $(\delta E)_{i}$ is randomly drawn for each system in a range $[-\mathrm{d} E,+\mathrm{d} E]$, assuming a uniform distribution. The normalized standard deviation of random mistuning, used to draw the Monte Carlo simulations results [9], is thus given by:

$\sigma(\mathrm{d} E)=\frac{2 \mathrm{~d} E}{\sqrt{12}}$ 


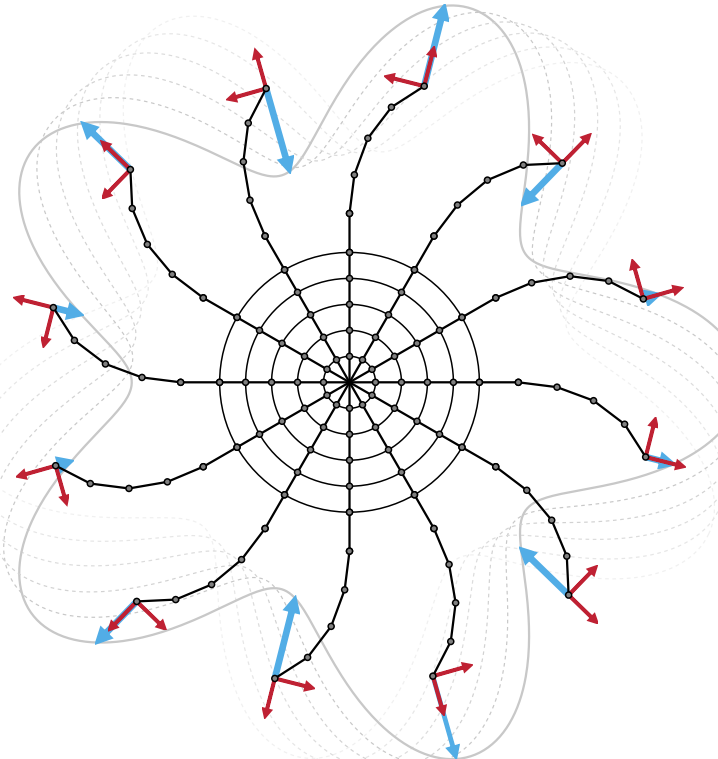

(a) linear configuration $\left(n_{d}=5\right)$ : circular load $(\rightarrow)$ and boundary dof $(\rightarrow)$

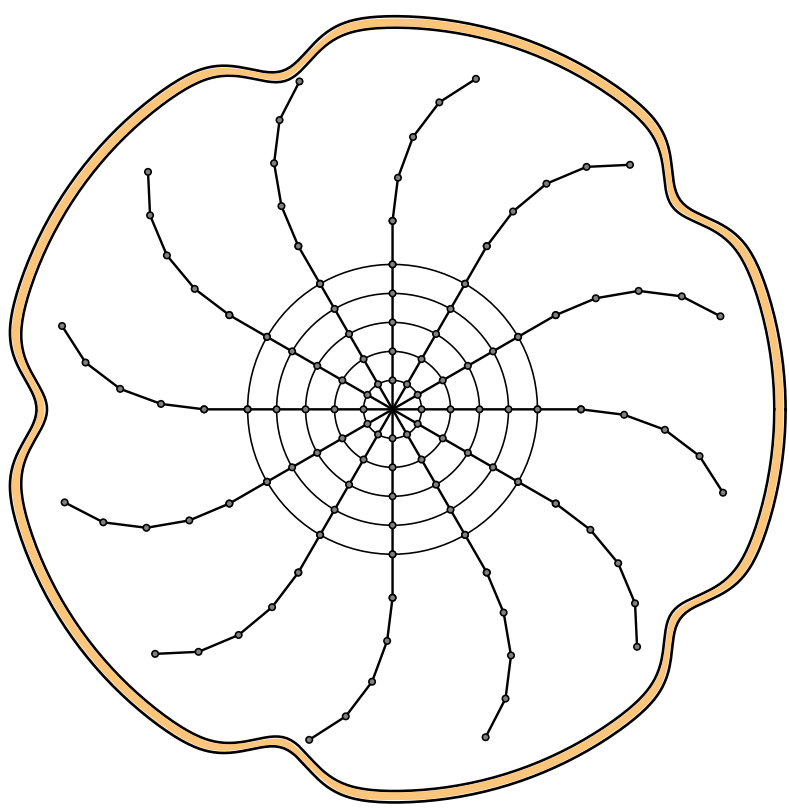

(b) nonlinear configuration $\left(n_{d}=5\right)$ : deformed casing $(\square)$

Figure 1. 2D bladed disk model.

The variation $\mathrm{d} E$ is set from $1 \%$ to $4 \%$, so that $\sigma(\mathrm{d} E)$ varies from $0.58 \%$ to $2.9 \%$, in agreement with levels of mistuning identified for modern manufacturing processes [17]. In the remainder, $\sigma(\mathrm{d} E)$ is called the mistuning level and is denoted $\sigma$ for the sake of brevity.

\section{Linear amplification}

Tuned and mistuned bladed disks are excited by a circular load $F_{\text {lin }}\left(\theta_{n}, t\right)$, see Fig. 1a. This load is a simplified representation of an aerodynamic forcing. Its application is limited to boundary dof in order to avoid any numerical artifact related to the modal reduction procedure:

$F\left(\theta_{n}, t\right)=F_{\max } \cos \left(\left(\theta_{n}-\Omega t\right) n_{d}\right)$

where $\theta_{n}$ stands for the angular position of the $n^{\text {th }}$-blade, $\Omega$ is the angular speed, and $F_{\max }$ is the amplitude of the forcing. It is here set to $F_{\max }=150 \mathrm{~N}$. Finally, $n_{d}$ represents the space frequency of this loading, thus related to the number of nodal diameters of the excitation. By choice, this notation is identical to the one used for the nonlinear excitation for the sake of later comparisons.

For each analyzed mistuned bladed disk, only the maximum vibration amplitude is retained over the frequency range of interest [9]. The ratio between the maximum vibration amplitude of the mistuned model and the one of the tuned model, classically called the mistuning amplification, will be referred to in the linear context as the linear amplification $A_{\text {lin }}$. Linear amplifications exhibited for the model have been validated with regards to the literature $[9,18]$ but are not detailed here for the sake of brevity.

\section{NONLINEAR ANALYSIS OF A MISTUNED BLADED DISK}

In order to illustrate every aspect of the proposed methodology, a random mistuning pattern with mistuning level $\sigma=1.7 \%$ is chosen, see Tab. 2. Key parameters and quantities of interest for the stochastic analysis are presented. 


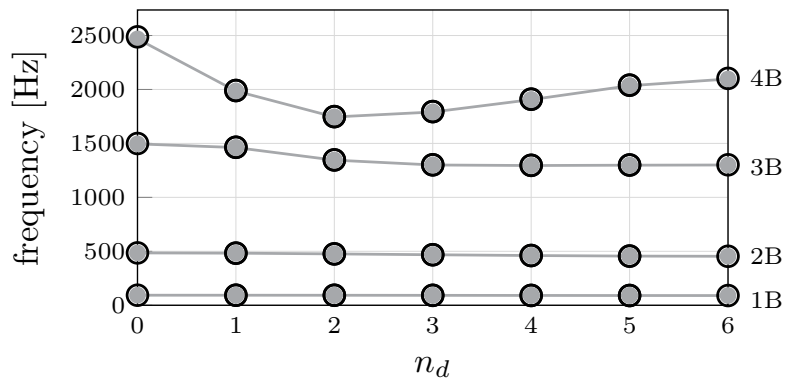

Figure 2. SAFE diagram for the ROM with $\eta=60(\mathrm{O})$ and for the full-FE model $(-\bullet)$.

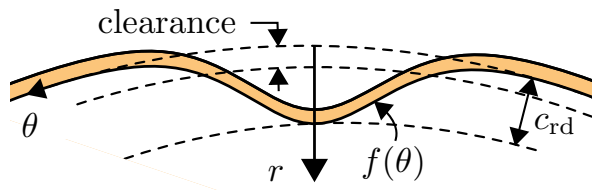

Figure 3. Casing deformation.

Because the validation of the overall numerical procedure was previously published [19], particular attention is here paid to: (1) the convergence of the simulations with regards to the 2D bladed disk reduction parameter $\eta$ and the time step $h$ and, (2) the robustness of the obtained results with respect to the different parameters of the model.

\section{Steady State Detection}

Contact simulations are carried out with a constant angular speed considering a deformed casing as depicted in Fig. 1b. To ensure that a steady state has been reached, time responses are thoroughly checked by means of an autocorrelation technique, with a focus on the tangential displacement of the blade-tip featuring the highest vibratory amplitudes. Simulations are first run over $0.5 \mathrm{~s}$, then the time response over the last three revolutions is compared to the time response over the previous three revolutions. Two criteria must then be satisfied to consider a steady state: (1) the correlation coefficient must be higher than the threshold value $c_{\text {cor }}$ and, (2) the relative error on the maximum amplitude must be lower than $\epsilon$. If any of these two criteria is not satisfied, the simulation is run over an additional ten revolutions until a steady state has been reached. In this study, a good compromise between accuracy and computation times was obtained by considering $c_{\text {cor }}=95 \%$ and $\epsilon=1 \%$. On average, steady states are reached for $t=0.96 \mathrm{~s}$.

\section{Interaction Maps}

Contact simulations are first performed over a wide angular speed range (from $10 \mathrm{~Hz}$ to $80 \mathrm{~Hz}$ ) on both the tuned and the mistuned bladed disk, for $n_{d}=4$, in order to better apprehend their vibratory behavior. The interaction maps thus generated are shown in Fig. 4.

For the tuned bladed disk, the highest amplitudes of vibration in Fig. 4a are witnessed for angular speeds in the vicinity of $34.45 \mathrm{~Hz}$ and $69 \mathrm{~Hz}$. These interactions respectively correspond to the crossing between the first bending modal family (1B) of the bladed disk and the fourth $\left(e_{o}=4\right)$ and second $\left(e_{o}=2\right)$ engine orders. It is visible that interactions are actually predicted for frequencies slightly higher (see, $\Delta_{1 \mathrm{~F}}$ in Fig. 4a) than those of the first bending modal family which is due to the contact stiffening phenomenon [11].

The interaction maps, as depicted in Fig. $4 \mathrm{~b}$, are key to define angular speed ranges of interest for the rest of this study. The influence of mistuning is noticeable, with both interaction areas spreading in a wider range $\delta f$ due to the frequency split phenomenon. Hence, the width of the angular speed range to consider to capture the maximum amplitudes of vibration depends on the mistuning pattern. Besides, for different values of $n_{d}$ different 
Table 2. Young's modulus per blade $\left(\times 10^{11} \mathrm{~Pa}\right)$ for the chosen random mistuning pattern.

\begin{tabular}{llllll}
\hline$E_{1}$ & 2.0701 & $E_{2}$ & 2.0627 & $E_{3}$ & 2.0695 \\
\hline$E_{4}$ & 2.1683 & $E_{5}$ & 2.0809 & $E_{6}$ & 2.1421 \\
\hline$E_{7}$ & 2.1164 & $E_{8}$ & 2.0166 & $E_{9}$ & 2.1256 \\
\hline$E_{10}$ & 2.0328 & $E_{11}$ & 2.0391 & $E_{12}$ & 2.1193 \\
\hline
\end{tabular}

Table 3. Angular speed range of interest for the mistuned bladed disk.

\begin{tabular}{lcccc}
\hline$n_{d}$ & 3 & 4 & 5 & 6 \\
\hline$\Omega_{\min }[\mathrm{Hz}]$ & 44.5 & 33.5 & 26.7 & 22 \\
\hline$\Omega_{\max }[\mathrm{Hz}]$ & 47.5 & 35.5 & 28.2 & 23 \\
\hline
\end{tabular}

interaction speeds are predicted. Stochastic analyses led to the angular speed range summed up in Tab. 3, with a focus kept on the intersection of the 1B modal family and the fourth engine order $e_{0}=4$.

\section{Nonlinear Forced Response Function}

Based on the angular speed ranges previously identified, contact simulations are run with an angular speed increment $\delta \Omega=0.05 \mathrm{~Hz}$. For each angular speed, the amplitude of the steady state is retrieved to draw the nonlinear forced response function (FRF) of the bladed disk. As an example, the FRF of both the mistuned bladed disk and the tuned bladed disk for $n_{d}=4$ are pictured in Fig. 5. Here again, it is noticeable that the frequency split $\delta f$ induced by mistuning significantly increases the angular speed range over which high amplitudes of vibration are found.

For each considered mistuning pattern, the quantity of interest is the maximum amplitude of vibration over the angular speed range [9]. The division of this quantity by the maximum amplitude of vibration of tuned bladed disk, found for $\Omega=34.45 \mathrm{~Hz}$, yields the nonlinear amplification, denoted $A_{\mathrm{nl}}$ in the following.

The intrinsically nonlinear nature of the investigated mechanical system requires that particular attention be paid to the obtained FRF in order to assess the robustness of the procedure. Indeed, it is likely that, due to unilateral contact constraints, the system exhibits an intricate dynamics featuring a variety of stable branches and

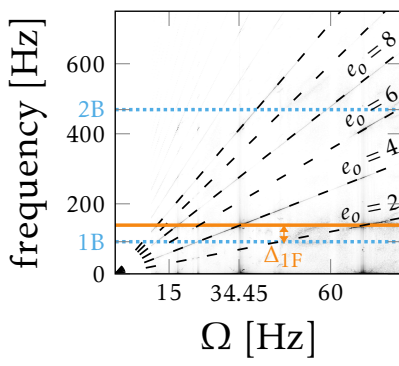

(a) tuned bladed disk

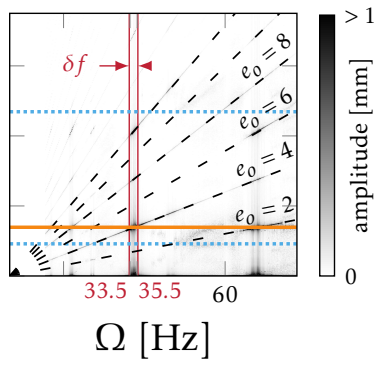

(b) mistuned bladed disk

Figure 4. Interaction maps for $n_{d}=4$, with engine order lines ( - - ). 
bifurcations, as have already been observed for 3D finite element models in similar contact configurations [20]. The FRF pictured in Fig. 5 does show signs of such occurrences, see, for instance, the sudden changes in amplitudes of vibration for the mistuned model (red area) and the tuned model (gray area). Yet a careful analysis of these jumps for various mistuning patterns reveals that they are never located in the vicinity of a peak and thus it is assumed that they do not impact the detection of the maximum of amplitude.

Finally, the obtained FRF for a small variations of key parameters such as the maximum casing deformation amplitude and the initial clearance are superimposed in Fig. 6. The fact that for small variations of the maximum casing deformation amplitude obtained FRF are simply slightly shifted highlights the robustness of the results.

\section{Time and Space Convergence}

In order to underline the convergence of the obtained results with respect to the time step $h$ of the time integration procedure, the nonlinear FRF obtained for different $h$ are plotted in Fig. 7a. FRF obtained for any value of $h>5 \times 10^{-5} \mathrm{~s}$ are almost perfectly superimposed. The slight differences that appear in the previously identified area for $\Omega \in[34.2,34.3] \mathrm{Hz}$ further suggest the co-existence of two stable branches of solution, as the variation of $h$ causes a small variation in the detection of the initial contact. In the following, all simulations are run considering $h=10^{-6} \mathrm{~s}$.

Space convergence is checked by varying the reduction parameter $\eta$ of the ROM. FRF obtained for different values of $\eta$, plotted in Fig. $7 \mathrm{~b}$, are almost perfectly superimposed from $\eta>36$. In the following, $\eta=60$ is considered so that the ROM contains a total of 84 dof: 24 boundary dof for contact management and 60 modal dof.

\section{NONLINEAR STOCHASTIC ANALYSIS}

Stochastic results depicting mistuning amplification for contact simulations are presented and discussed in this section. Contact simulation parameters are recalled in Tab. 4 . After a careful assessment of the stochastic result convergence, predicted nonlinear and linear amplifications are compared for different values of $n_{d}$ and mistuning levels. In particular, a subsection is dedicated to the configuration $n_{d}=4$ with a cross-analysis based on all the mistuning patterns used for contact simulations.

\section{Convergence}

Due to the nonlinear nature of each contact simulation, there is no empirical guideline on how many samples are required in order to reach convergence with Monte Carlo simulations. On the one hand, it is a prerequisite to reach convergence so that presented results are relevant, but on the other hand the high cost of these nonlinear simulations in terms of computation times (the nonlinear FRF for each sample requires about one hour calculation on a standard i7 processor based PC) makes it difficult to reach the number of samples usually considered in a linear and less expensive context [1]. Accordingly, convergence is here carefully assessed both qualitatively and quantitatively in order to ensure efficient Monte Carlo simulations.

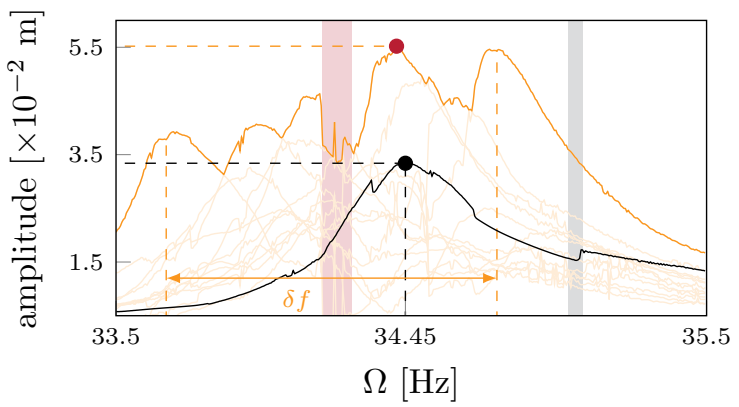

Figure 5. FRF for $n_{d}=4$ : mistuned $(-)$ and tuned bladed disk $(\boldsymbol{-})$. 


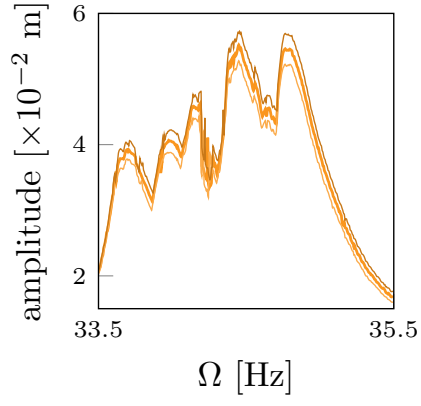

(a)

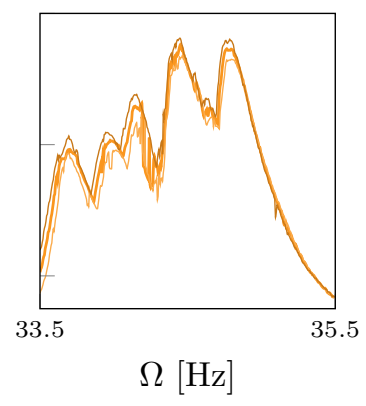

(b)

Figure 6. FRF for varying casing deformation amplitude (a) and initial clearance (b): $-0.05 \mathrm{~mm}(-)$, reference case $(-)$, $+0.05 \mathrm{~mm}(-)$.

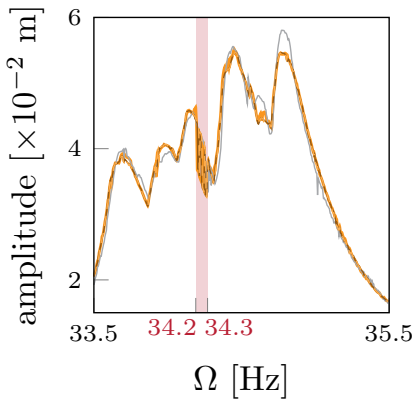

(a)

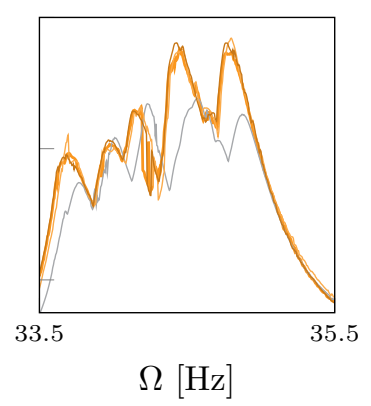

(b)

Figure 7. Time (a) and space (b) convergence on the FRF for $n_{d}=4:(-)$ for $h=5 \times 10^{-5} \mathrm{~s}$ and $\eta=12,(-)$ for $h=5 \times 10^{-6} \mathrm{~s}$ and $\eta=36,(-)$ for $h=10^{-6}$ s and $\eta=60,(-)$ for $h=5 \times 10^{-7}$ s and $\eta=96$.

\section{Qualitative Assessment}

Qualitative convergence is checked through the stabilization of the mean of amplification with regards to the number of considered samples, for each percentile and each mistuning level $\sigma$, as shown in Fig. 8. Semi-log scales are used to emphasize any variation of the results, thus avoiding overestimating convergence rates. It is observed that convergence is reached for 2000 samples per mistuning level for $n_{d}=6$ while 3000 samples are required for $n_{d}=3$ and 5. Finally, 4000 samples must be considered for $n_{d}=4$.

\section{Quantitative Assessment}

Quantitative convergence is checked using the law of large numbers [21] and the central limit theorem [21] on the standard error of the mean. These tools allow for the definition of a convergence error:

$\operatorname{error}\left(p_{s}\right)=Z \sqrt{\frac{V\left(p_{s}\right)}{s}}$

where $p_{s}$ represents the percentile values for $s$ samples, $V$ is the variance, thus making $\sqrt{\frac{V\left(p_{s}\right)}{s}}$ the standard error of the mean, and $Z$ is the confidence coefficient determined with the right-hand side standard normal Z-table. Classically, confidence level values used are $95 \%, 99 \%$ and $99.5 \%$ [21]. In this study, a $99.5 \%$ confidence 
level is considered to ensure high accuracy results, hence $Z=2.81$. The variance is estimated with its unbiased estimator [21]:

$V\left(p_{s}\right)=\frac{1}{s-1} \sum_{i=1}^{s}\left|p_{s}-\overline{p_{s}}\right|^{2}$

where $\overline{p_{s}}$ is the mean of $p_{s}$. It should be noted that the defined error advantageously does not depend on the mean of all the samples. Convergence errors with regards to the number of samples for various nodal diameters are depicted in Fig. 9. It is assumed that results are converged for error $\left(p_{s}\right)<0.5 \%$. Monte Carlo simulations for $n_{d}=6$ are the fastest to converge, with a convergence criteria reached as early as 500 samples per mistuning level, while simulations for $n_{d}=3$ require a minimum of 2000 samples.

\section{Conclusion}

Both quantitative and qualitative analyses of the carried out stochastic simulations underline that, in the worst configuration, reaching convergence requires 4000 samples. Interestingly, it thus seems that the contact constraints imposed on the bladed disk allow for a faster stochastic convergence than what is found in the literature for linear investigations where around 10000 samples are usually considered [1], a value that is indeed found to yield convergence for linear simulations on the model used in this study. This may be a consequence of the reduced admissible domain for vibrations in the radial direction due to contact. In order to ensure fully converged results, 5000 samples are used in this study for each point $\left(n_{d}, \sigma\right)$ of the investigated parameter space.

\section{Vibration Amplification}

\section{Comparison to Linear Results}

Mistuning linear and nonlinear amplifications (denoted respectively $A_{\operatorname{lin}}$ and $A_{\mathrm{nl}}$ ) for various $n_{d}$ and $\sigma$ are drawn in Fig. 10. The comparative analysis of these two types of amplification highlight the strong influence of both $n_{d}$ and $\sigma$. Indeed, while nonlinear amplifications are overall much higher than linear amplifications (see $n_{d}=3,4$ and $5)$, it is noticeable that nonlinear amplifications are predicted below linear amplifications for $n_{d}=6$. With respect to $\sigma$, the gap between $A_{\operatorname{lin}}$ and $A_{\mathrm{nl}}$ is maximum for lower $\sigma$ for $n_{d}=3$ and 5 while it monotonically increases with $\sigma$ for $n_{d}=4$ and 6 . It is worth noting that these trends are consistent throughout percentiles.

Nonlinear amplifications are the largest for $n_{d}=4$ : they are plotted in Fig. 10d for all percentiles along corresponding linear amplifications. Percentile 50 for nonlinear amplifications is almost superimposed with percentile 99 for linear amplifications, and becomes even higher for $\sigma=2.3 \%$. These results suggest a much higher sensitivity of the bladed disk model to mistuning when contact events occur. Maximum amplifications in a linear framework $\left(57 \%\right.$ for percentile 99 , as $\left.A_{\text {lin }}=1.57\right)$ are indeed significantly increased when structural contacts occur ( $75 \%$ for percentile 99 , as $\left.A_{\mathrm{nl}}=1.75\right)$.

These results also underline that numerical predictions made on the vibration behavior of a tuned bladed disk undergoing structural contacts are robust with respect to small mistuning. Thus, interactions identified for the tuned case are also interactions for mistuned bladed disks with potentially much higher amplitudes of vibration.

Table 4. Contact simulation parameters.

\begin{aligned} & \hline time step $h=1 \cdot 10^{-6} \mathrm{~s} \\ &$\hline reduction parameter $\eta=60 \\ &$\hline casing initial clearance $0.25 \mathrm{~mm} \\ &$\hline blade/casing coefficient of friction 0.15 \\ & \hline\end{aligned}




\section{Analysis}

The linear amplification $A_{\text {lin }}$ variation with respect to $n_{d}$ is explained mostly in two ways in the literature: (1) the forcing frequency may correspond to a veering region [22] or, (2) the forcing frequency may excite closely spaced blade dominated modes, identified on the tuned system [18]. As underlined in the SAFE diagram, see Fig. 2, the proposed model does not feature veering regions around the forcing frequency (corresponding to the 1B modal family), but modes of the 1B family are indeed closely spaced.

Yet, to the best of the authors' knowledge, there is no mention of the influence of $n_{d}$ on nonlinear amplifications in the literature. In order to explain the observations made in the previous section, it is proposed to put them into perspective in light of the maximum amplitudes of vibration given in Fig. 11. For all degrees of mistuning $\sigma$, amplitudes of vibration strongly increase when looking at percentiles 99 of the stochastic results. However, it is noticeable that for $n_{d}=3$ and $n_{d}=6$, for which tuned amplitudes of vibration are the highest, mistuned amplitudes of vibrations are clustered within a very narrow range located around $65 \mathrm{~mm}$.

Conversely, values of $n_{d}$ for which the tuned system features lowest amplitudes of vibration $\left(n_{d}=4\right.$ and $\left.n_{d}=5\right)$ yield similar trends as increasing mistuning degree leads to higher amplitudes of vibration. Contrary to the linear configuration where there is no constraint on the bladed disk vibration, contact with the surrounding casing does constrain the bladed disks amplitudes of vibration. Thus, it appears that clustered results predicted for $n_{d}=3$ and $n_{d}=6$ are essentially due to an over-constrained domain of vibration: mistuning amplification is here mitigated by the rigid casing.

In that sense, the proposed computation of amplifications as a ratio of displacements at the blade-tips may provide an optimistic representation of what is really happening for $n_{d}=3$ and $n_{d}=6$. Though a more in-depth analysis of these two configurations goes beyond the scope of this study, it seems likely that an amplification based on strain energy would underline the detrimental effect of mistuning for these two configurations.

In the end, it seems that for the proposed model and contact scenario, the value $n_{d}=4$ provides the most representative results: because predicted amplitudes of vibration for the tuned case are lower, it is possible to fully capture their increase due to mistuning. For this reason, the focus is made on the configuration $n_{d}=4$ in the following.

Cross-Analysis for $n_{d}=4$

The 5000 samples considered for contact simulations with $n_{d}=4$ and for each mistuning level $\sigma$ are subjected to the linear forcing defined in Eq. (5). Their amplifications are thus obtained in both linear and nonlinear contexts. These values are depicted in the $\left(A_{\mathrm{nl}}, A_{\mathrm{lin}}\right)$ plane, see Fig. 12. Cumulative densities, calculated from the highest to the lowest density regions, are preferred to a scatter plot in order to get a clearer view of denser areas.

Overall, the majority of mistuning patterns features $A_{\mathrm{nl}}>A_{\operatorname{lin}}$ : the majority of data points are indeed located above the line $A_{\mathrm{nl}}=A_{\text {lin }}$. This trend is reinforced as the mistuning level $\sigma$ increases. More precisely, for $\sigma=0.6 \%$,

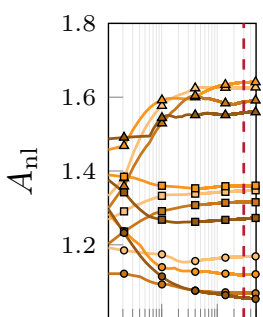

$10^{2} 10^{3}$

$s$

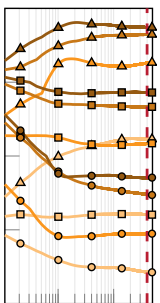

$10^{2} \quad 10^{3}$

$s$

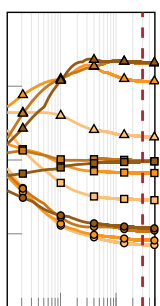

$10^{2} \quad 10^{3}$

$s$

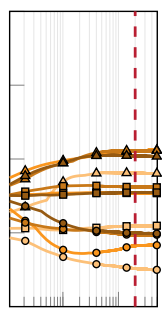

$10^{2} \quad 10^{3}$

$s$
(a) $n_{d}=3$
(b) $n_{d}=4$
(c) $n_{d}=5$
(d) $n_{d}=6$

Figure 8. Qualitative convergence assessment on percentile 1 ( ० ), 50 ( $\square$ ) and $99(\Delta)$ for $\sigma=0.6 \%(-), 1.2 \%(-), 1.7 \%(-)$ and $2.3 \%(-)$. 
the nonlinear amplification is higher than the linear amplification for $82 \%$ of the samples. This number grows to $96 \%$ for $\sigma=2.3 \%$. It is noticeable that mistuning patterns with highest values of $A_{\mathrm{nl}}$ spread along a wide range of $A_{\text {lin }}$ values. For instance, for $\sigma=1.7 \%$, mistuning patterns above percentile 99 of $A_{\mathrm{nl}}$ values correspond to $A_{\text {lin }} \in[1,1.7]$. These results thus underline that there is no correlation between mistuning patterns featuring high linear amplifications and mistuning patterns featuring high nonlinear amplifications. Accordingly, mitigating linear amplifications and nonlinear amplifications may call for distinct design strategies and existing techniques available in the literature $[3,4,23]$ should also be assessed in a structurally nonlinear context.

\section{CONCLUSION}

This paper presents a first insight on the influence of small mistuning on the amplitudes of vibration following blade-tip/casing contact events, by means of numerical simulations on a $2 \mathrm{D}$ phenomenological bladed disk. The used numerical procedure relies on numerical time integration with a Lagrange multiplier-based contact algorithm. Such procedure, dedicated to the prediction of blade-tip/casing contact induced interactions, has been previously validated with respect to both experimental set-ups and academic test cases. Mistuning is accounted for as a variation of blades Young's modulus. Attention is paid to the validation of the proposed numerical strategy, namely through its convergence with respect to key parameters. It is also underlined that presented results are robust with respect to simulation parameters including the casing deformation amplitude and the blade-tip/casing clearance.

By means of Monte Carlo simulations, linear and nonlinear amplifications-defined as the ratio between the maximum amplitude of vibration of mistuned structures and the one of the tuned model-are analyzed comparatively for different configurations. In comparison to amplifications predicted in a linear context, presented results suggest that mistuning may lead to even higher amplifications when contacts are accounted for. These results are observed for various configurations and several mistuning levels. Also, this study confirms that predicted interactions on a tuned bladed disk are robust with respect to small mistuning: all predicted critical speeds on a tuned blade disk are also found critical for mistuned structures.

The carried out cross-analysis of linear and nonlinear amplifications for a given contact configuration underlines that there is no correlation between mistuning patterns exhibiting high amplifications in a context or another. As a matter of fact, nonlinear amplifications are predicted for mistuned patterns featuring low to high linear amplifications. As a consequence, results obtained with this phenomenological bladed disk hint that design strategies to mitigate mistuning amplifications in a linear context may not be well suited for mitigating nonlinear amplifications. Existing techniques available in the literature to mitigate linear amplifications [3, 4, 23] should thus be assessed in a structurally nonlinear context.

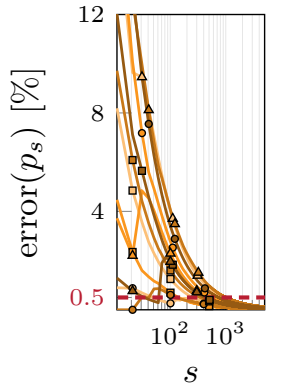

(a) $n_{d}=3$

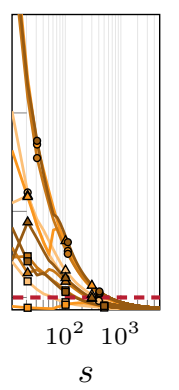

(b) $n_{d}=4$

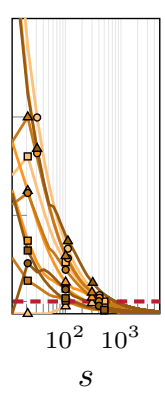

(c) $n_{d}=5$

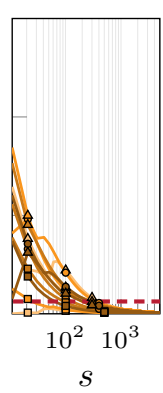

(d) $n_{d}=6$

Figure 9. Quantitative convergence assessment on percentile 1 ( ○), 50 ( $\square)$ and $99(\triangle)$ for $\sigma=0.6 \%(-)$, $1.2 \%(-)$, $1.7 \%(-)$ and $2.3 \%(-)$. 


\section{Acknowledgment}

This research was supported by the Fonds de recherche du Québec - Nature et technologies (FRQ-NT) and the Natural Sciences and Engineering Research Council of Canada (NSERC).

\section{References}

[1] M. P. Castanier, C. Pierre, Modeling and Analysis of Mistuned Bladed Disk Vibration: Status and Emerging Directions, Journal of Propulsion and Power 22 (2) (2006) 384-396. do i : 10.2514/1. 16345.

[2] Y.-J. Chan, D. J. Ewins, Management of the variability of vibration response levels in mistuned bladed discs using robust design concepts. Part 2: Tolerance design, Mechanical Systems and Signal Processing 24 (8) (2010) 2792-2806. do i:10.1016/ j ymssp.2010.06.005.

[3] M. P. Castanier, C. Pierre, Using Intentional Mistuning in the Design of Turbomachinery Rotors, AIAA Journal 40 (10) (2002) 2077-2086. do i : 10 .2514/3. 15298.

[4] Y. Han, R. Murthy, M. P. Mignolet, J. Lentz, Optimization of Intentional Mistuning Patterns for the Mitigation of the Effects of Random Mistuning, Journal of Engineering for Gas Turbines and Power 136 (6) (2014) 1-9. doi:10.1115/1.4026141.

[5] F. Figaschewsky, A. Kühhorn, B. Beirow, J. Nipkau, T. Giersch, B. Power, Design and analysis of an intentional mistuning experiment reducing flutter susceptibility and minimizing forced response of a jet engine fan, in: ASME Turbo Expo, 2017, pp. V07BT36A020-V07BT36A020. doi : 10. 1115/GT2017-64621.

[6] R. Corral, O. Khemiri, C. Martel, Design of mistuning patterns to control the vibration amplitude of unstable rotor blades, Aerospace Science and Technology 80 (2018) 20-28. do i : 10 . 1016/ j . ast.2018.06.034.

[7] C. Joannin, B. Chouvion, F. Thouverez, J.-P. Ousty, M. Mbaye, A nonlinear component mode synthesis method for the computation of steady-state vibrations in non-conservative systems, Mechanical Systems and Signal Processing 83 (15) (2017) 75-92. do i : 10.1016/ j ymssp.2016.05.044.

[8] B. Beirow, F. Figaschewsky, A. Kühhorn, A. Bornhorn, Modal Analyses of an Axial Turbine Blisk With Intentional Mistuning, Journal of Engineering for Gas Turbines and Power 140 (1) (2018) 012503. doi: $10.1115 / 1.4037588$.

[9] G. Óttarsson, C. Pierre, On the effects of interblade coupling on the statistics of maximum forced response amplitudes in mistuned bladed disks, AIAA 1494-CP (1995) 3070-3078. do i : 10 .2514/6 . 1995-1494.

[10] R. Rajasekharan, E. Petrov, Analysis of Deformation of Mistuned Bladed Disks With Friction and Random Crystal Anisotropy Orientation Using Gradient-Based Polynomial Chaos Expansion, in: ASME Turbo Expo, 2018, p. V07CT35A029. doi: 10.1115/GT2018-76566.

[11] G. Jacquet-Richardet, M. Torkhani, P. Cartraud, F. Thouverez, T. Nouri Baranger, M. Herran, C. Gibert, S. Baguet, P. Almeida, L. Peletan, Rotor to stator contacts in turbomachines. Review and application, Mechanical Systems and Signal Processing 40 (2) (2013) 401-420. do i : 10.1016/ j .ymssp.2013.05.010.

[12] M. Legrand, C. Pierre, P. Cartraud, J. P. Lombard, Two-dimensional modeling of an aircraft engine structural bladed disk-casing modal interaction, Journal of Sound and Vibration 319 (1-2) (2009) 366-391. doi: 10.1016/ j. jsv.2008.06.019.

[13] N. Salvat, A. Batailly, M. Legrand, Two-dimensional modeling of unilateral contact-induced shaft precessional motions in bladed-disk/casing systems, International Journal of Non-Linear Mechanics 78 (2016) 90-104. doi:10.1016/j.ijnonlinmec.2015.10.001.

[14] R.-R. Craig, M. C. C. Bampton, Coupling of Substructures for Dynamics Analyses, AIAA Journal 6 (7) (1968) 1313. doi: $10.2514 / 3.4741$. 
[15] N. J. Carpenter, R. L. Taylor, M. G. Katona, Lagrange Constraints for Transient Finite Element Surface Contact, International Journal for Numerical Methods in Engineering 32 (1) (1991) 103-128. doi:10.1002/nme. 1620320107.

[16] T. Klauke, A. Kühhorn, B. Beirow, M. Golze, Numerical Investigations of Localized Vibrations of Mistuned Blade Integrated Disks (Blisks), Journal of Turbomachinery 131 (3) (2009) 031002. do i : 10 .1115/1.2985074.

[17] E. Capiez-Lernout, C. Soize, J.-P. Lombard, C. Dupont, E. Seinturier, Blade manufacturing tolerances definition for a mistuned industrial bladed disk, in: ASME Turbo Expo, Vol. 6, 2004, pp. 307-316. doi:10.1115/ GT2004-53356.

[18] J. Judge, C. Pierre, O. Mehmed, Experimental Investigation of Mode Localization and Forced Response Amplitude Magnification for a Mistuned Bladed Disk, Journal of Engineering for Gas Turbines and Power 123 (4) (2001) 940-950. doi : 10.1115/1.1377872.

[19] A. Batailly, M. Legrand, A. Millecamps, F. Garcin, Numerical-Experimental Comparison in the Simulation of Rotor/Stator Interaction Through Blade-Tip/Abradable Coating Contact, Journal of Engineering for Gas Turbines and Power 134 (8) (2012) 082504. doi : 10.1115/1.4006446.

[20] A. Batailly, M. Legrand, A. Millecamps, F. Garcin, Conjectural Bifurcation Analysis of the Contact-Induced Vibratory Response of an Aircraft Engine Blade, Journal of Sound and Vibration 348 (21) (2015) 239-262. doi:10.1016/j.jsv.2015.03.005.

[21] C. Graham, D. Talay, Stochastic Simulation and Monte Carlo Methods, Springer, 2013.

[22] A. G. Joshi, B. I. Epureanu, Reduced Order Models for Blade-To-Blade Damping Variability in Mistuned Blisks, Journal of Vibration and Acoustics 134 (2012) 051015. doi:10.1115/1.4006880.

[23] B. Beirow, F. Figaschewsky, A. Kühhorn, A. Bornhorn, Vibration analysis of an axial turbine blisk with optimized intentional mistuning pattern, Journal of Sound and Vibration 442 (3) (2019) 11-27. do i : 10. 1016/ j. jsv. 2018.10.064. 


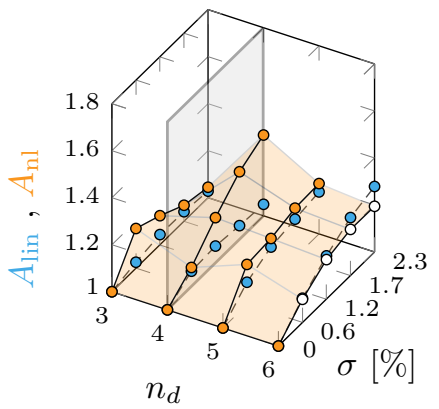

(a) percentile 1

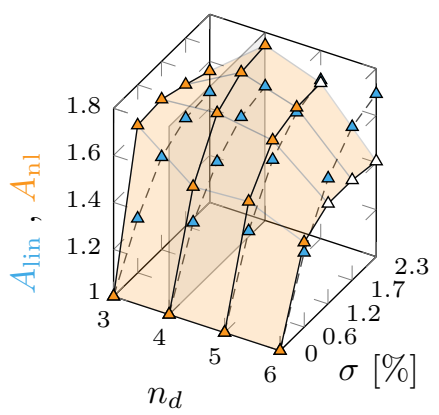

(c) percentile 99

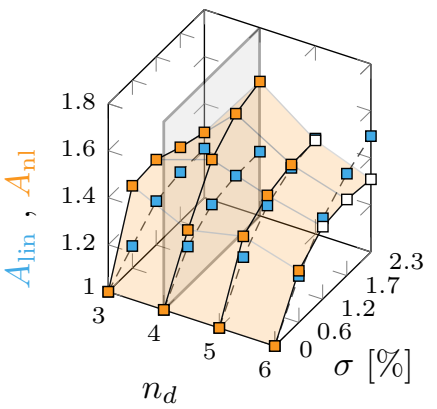

(b) percentile 50

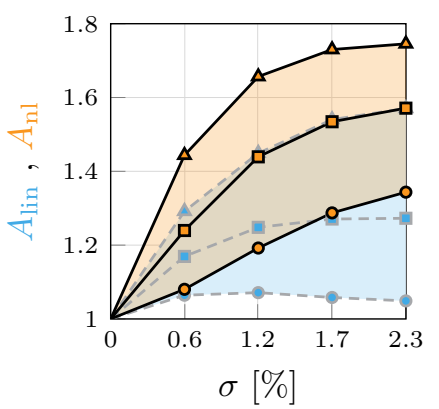

(d) all percentiles for $n_{d}=4$

Figure 10. Nonlinear $(\square)$ and linear $(\square)$ amplification for different $n_{d}$, with percentiles $1(\circ), 50(\square)$ and $99(\Delta)$.

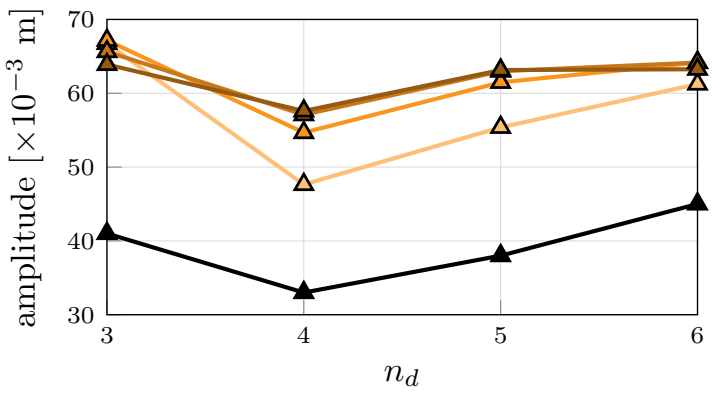

Figure 11. Maximum amplitudes of vibration for the tuned ( $\boldsymbol{-}-$ ) and mistuned bladed disks (percentile 99$)$ for $\sigma=0.6 \%(\boldsymbol{\Delta})$, $\sigma=1.2 \%(\boldsymbol{\Delta}), \sigma=1.7 \%(\boldsymbol{\Delta})$ and $\sigma=2.3 \%(\boldsymbol{\Delta})$ during contact simulations. 


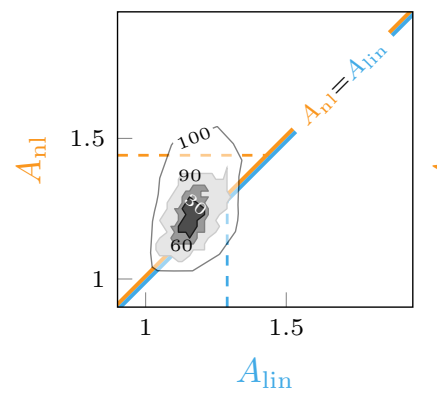

(a) $\sigma=0.6 \%$

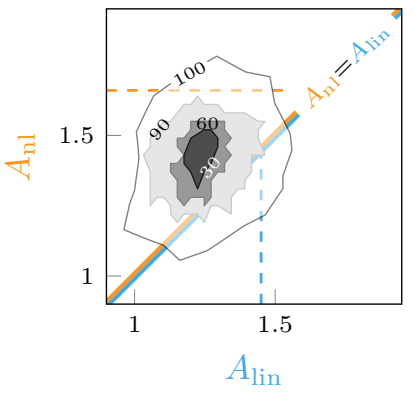

(b) $\sigma=1.2 \%$

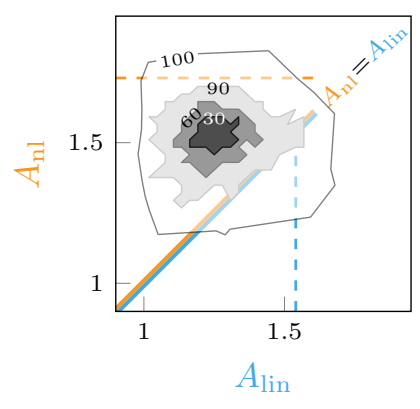

(c) $\sigma=1.7 \%$

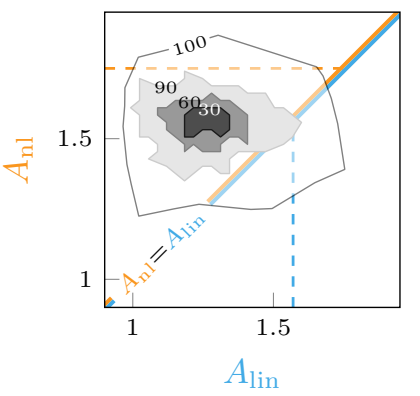

(d) $\sigma=2.3 \%$

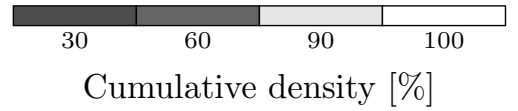

Cumulative density [\%]

Figure 12. Cross-analysis of mistuning amplification, for $n_{d}=4$, with percentiles 99 for nonlinear $(=-)$ and linear $(-=)$ simulations. 